\title{
Fuzzy Adaptive Controller Design for Double- pendulum Tower Crane with Distribute Mass Payload
}

\section{Zheng Sun}

Nanjing Tech University

Huimin Ouyang ( $\square$ ouyang1982@njtech.edu.cn )

Nanjing Tech University https://orcid.org/0000-0002-5278-9563

\section{Research Article}

Keywords: Underactuated systems , Tower cranes , Distributed mass payload , Antiswing control , Fuzzy adaptive control

Posted Date: October 20th, 2021

DOI: https://doi.org/10.21203/rs.3.rs-911408/v1

License: (c) (i) This work is licensed under a Creative Commons Attribution 4.0 International License.

Read Full License 


\title{
Fuzzy Adaptive Controller Design for Double-pendulum Tower Crane with Distribute Mass Payload
}

\author{
Zheng Sun • Huimin Ouyang
}

Received: date / Accepted: date

\begin{abstract}
As a kind of high-efficiency transportation tools, tower cranes are widely used in construction site. With the increasing volume and mass of payload being transported, the researches of distributed mass payload (DMP) problems have been paid more and more attentions. However, most of the existing control algorithm$\mathrm{s}$ designed for the concentrated mass payload (CMP) are not enough to meet the needs of actual production. The difference between DMP and CMP is mainly manifested in that the remaining payload swing caused by inertial torque of DMP cannot be effectively suppressed, which leads to safety hazards. In addition, due to the different working environment, accurate system parameters (such as mechanical frictions, air frictions ) are hard to obtain, which leads to errors in their positioning. To solve the above issues, first, we establish mathematical model of a double-pendulum tower crane with distributed mass payload (DTCDMP) and carry out dynamic analysis. Then we propose a fuzzy adaptive control method, which has a good tracking effect against external disturbances and parameter uncertainties, and the method can achieve accurate positioning and effective anti-swing. Then, the Lyapunov technology and LaSalle's invariance principle are used to rigorously prove the stability of the system. Finally, on the basis of tracking the S-shaped trajectories, the effectiveness and robustness of the proposed controller are verified through multi-group comparative experiments.
\end{abstract}

Z. Sun, H. Ouyang (Corresponding author)

College of Electrical Engineering and Control Science, Nanjing Tech University, No.30, Puzhu Road(s), Nanjing, 211816, China

Zheng Sun

E-mail: 202061206081@njtech.edu.cn

E-mail: ouyang1982@njtech.edu.cn
Keywords Underactuated systems - Tower cranes . Distributed mass payload · Antiswing control · Fuzzy adaptive control

\section{Introduction}

With the development of modern industry, the crane system has been widely used in various production departments such as factories, mines, construction sites, etc., due to its advantages of fewer actuators and simple structure [1][2]. However, as an under-actuated system, the number of independent inputs of a crane are less than the number of degrees of freedom, and there is usually a high degree of coupling between the system states (jib rotation, trolley movement and cargo swing). This also makes eliminate the swing angles while ensuring its positioning (jib rotation and trolley displacement) become a very challenging problem during operation.

In recent decades, the control problem for cranes has attracted attention by scholars from various countries. In this regard, a series of control algorithms have also been proposed, such as input shaping method [3]-[5], trajectory planning method [6][7], partial feedback linearization [8], nonlinear coupling control [9], adaptive control [10][11], sliding mode control [12]-[14], neural network [15][17] and other control algorithms. Through careful analysis, it is found that the above literature is mainly for the crane system with single pendulum effect. However, in practical applications, the mass of the hook cannot be ignored. Therefore, it is easier to show the double-pendulum effect during the swing process. In addition, we also need to consider factors such as payload volume and shape. In this case, the payload has distributed mass characteristics. the swing phenomenon of DMP is more complicated. 
For this reason, scholars have also put forward some effective schemes and achieved certain results. Jaafar et al. proposed a model reference command shaping method for the payload multi-modal vibration control problem in the bridge crane system. Compared with the multi-modal zero vibration shaper and the derivative zero vibration shaper, it has higher robustness [18]. Lu et al. proposed an enhanced coupling adaptive control method for the variable rope length double-pendulum bridge crane system, and the effectiveness of the method was verified by platform experiments [19]. In order to realize the trolley positioning and payload swing suppression in the double-pendulum bridge crane system, Ouyang et al. proposed a controller based on energy shaping [20]. Giacomelli et al. aimed at the residual swing of the payload in the double-pendulum bridge crane system. An input-output inverse control method is proposed, and the effectiveness of the method is verified by simulation and experiments [21]. Sun et al. proposed an energy/passivity-based adaptive nonlinear controller for the double-pendulum bridge crane system, and use the experiment verified the effectiveness of the method [22]. Chen et al. also proposed an energyoptimized controller for the dual-swing bridge crane system. This method not only realizes the positioning and swing suppression of the trolley, but also considers the constraints of the system state and control variables. Finally, a comparative experiment verifies the effectiveness of method [23]. Aiming at the horizontal oscillation of the two-dimensional plant after the distributed mass payload reaches the target position, Wang et al. proposed an optimized and improved optimal anti-sway control method based on the system response under the action of a first-order low-pass filter to achieve higher hook oscillation. The faster suppression of distributed mass payload oscillation is verified by experiment and simulation [24].

Through a large amount of literature analysis and comparison, some important issues still remain to be solved.

1. Many control methods are designed for the crane system with CMP. However, there is no specific analysis for DTCDMP. Therefore, the effectiveness of the existing control algorithms needs to be studied in detail.

2. The design of most controllers for double-pendulum tower cranes are based on linear model. While encountering external disturbances, the state variables are easy to deviate from the equilibrium position, and its stability cannot be guaranteed.

3. Most of the existing control algorithms require accurate parameter information, and it is difficult to ensure the robustness to uncertain parameters.
In response to the above problems, the main contributions of this paper are as follows:

1. The dynamic model of the DTCDMP is established and analyzed.

2. Aiming at the complex dynamic characteristics of DTCDMP, a fuzzy adaptive controller is proposed without linearization. It can adjust controller parameters online to ensure accurate positioning and fast sway elimination. Therefore, it has more excellent control performance under different parameters.

3. The Lyapunov technique and LaSalle's invariance principle are used to prove its stability, and through comparative experiment analysis, the controller has good anti-swing performance.

The remaining structure of this paper is as follows: In Section 2, the mathematical model of DTCDMP is established. Then, Section 3 describes the design of the proposed controller. The adaptive controller is designed according to the energy equation, in order to enhance the real-time control, a fuzzy controller is added, and the two are combined into the proposed controller to further improve the positioning and sway function. Section 4 introduces the self-built tower crane platform and the discussion and analysis of the experimental results, which proves the superiority of the proposed controller. Section 5 summarizes the main work of this paper.

\section{Problem Statement}

\subsection{Tower Crane Modeling}

In order to clearly express the physical dynamic structure and operating mechanism of the DTCDMP, the model diagram is shown in Fig. 1. It is roughly composed of the jib, base, mast, trolley, hook and DMP. The Lagrange's equation of motion is used to establish its mathematical dynamic relationship, and the results is as follows:

$$
M(q) \ddot{q}+C(q, \dot{q}) \dot{q}+G(q)=U-F_{s}-D
$$

where $M(q)$ is system inertia matrix, $C(q, \dot{q})$ is referred to as the centripetal-Coriolis matrix, $G(q)$ is the gravity effects vector, $U$ is the control input vector, $F_{s}$ and $D$ are the system disturbances, $q(t)$ is the state variable of the system. For ease of reading, the specific content is included in Appendix A.

Considering the actual operation of the crane and the convenience of the controller design and the proof of stability, we make the following reasonable assumptions. 


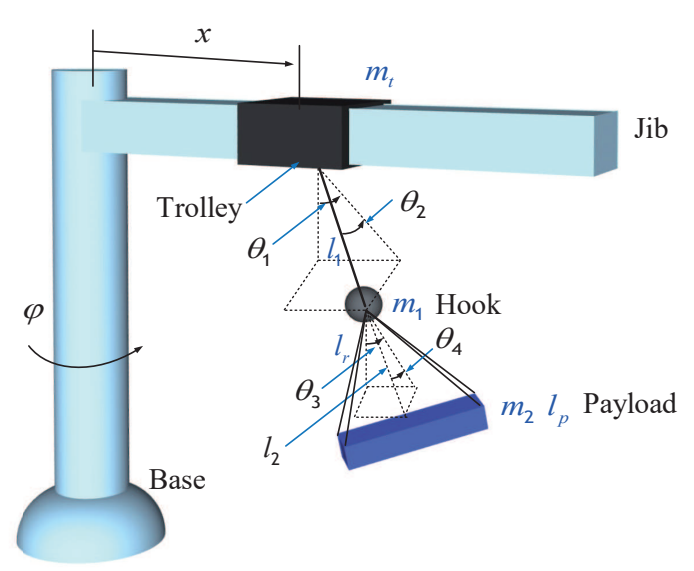

Fig. 1 Structure diagram of tower crane with DMP.

Assumption 1: Ropes and rigging can be regarded as a massless rigid link.

Assumption 2: During transportation, the swing angles of the hook and payload are:

$\theta_{i} \in\left(-\frac{\pi}{2}, \frac{\pi}{2}\right), i=1, \ldots, 4$.

\subsection{Control Objective}

The control objectives of the tower crane system include fast and accurate positioning of the trolley and jib, and effective suppression of payload swing. In order to achieve the entire control goal, it is defined as follows:

$\lim _{t \rightarrow \infty} \chi=\chi_{r}$

where $\chi=\varphi$ or $x, \chi_{r}$ is the target position (jib/trolley).

$\lim _{t \rightarrow \infty} \theta_{i}=0, i=1, \ldots, 4$.

At the same time, the trolley and jib operation meet the following reference trajectories,

case 1: $t \geq t_{\chi d}$

$\chi_{r}(t)=\chi_{d}, \dot{\chi}_{r}(t)=0, \ddot{\chi}_{r}(t)=0$,

case 2: $0<t<t_{\chi d}$

$\chi_{r}, \dot{\chi}_{r}, \ddot{\chi}_{r} \in L_{\infty}$,

case 3: $t=0$

$\chi_{r}(0)=\chi_{0}, \dot{\chi}_{r}(0)=0, \ddot{\chi}_{r}(0)=0$

where $\chi_{0}$ is the initial position (jib/trolley).

\section{Main Results}

In this section, aiming at DTCDMP, a fuzzy adaptive controller is proposed. Then we use Lyapunov technique and LaSalle's invariance principle to prove its stability.

\subsection{Adaptive Controller Design}

The total energy of the DTCDMP system is the sum of its kinetic and potential energy. The mathematical expression is:

$$
\begin{aligned}
E & =\frac{1}{2} \dot{q}^{T} M \dot{q}+\left(m_{1}+m_{2}\right) g l_{1}\left(1-C_{1} C_{2}\right) \\
& +m_{2} g l_{2}\left(1-C_{3} C_{4}\right)
\end{aligned}
$$

It is worth mentioning that, for ease of reading, let $\sin \left(\theta_{i}\right) \triangleq S_{i}, \cos \left(\theta_{i}\right) \triangleq C_{i}(\mathrm{i}=1, \ldots, 4$. $)$ and $l_{2}^{2}=l_{r}^{2}-\frac{l_{p}^{2}}{4}$ in the following sections.

The energy function is $E$ derived with respect to time, and $\dot{E}$ is expressed as follows:

$$
\begin{aligned}
& \dot{E}=\ddot{q}^{T} M \dot{q}+\frac{1}{2} \dot{q}^{T} \dot{M} \dot{q}+\left(m_{1}+m_{2}\right) g l_{1}\left(S_{1} C_{2} \dot{\theta}_{1}\right. \\
&\left.+C_{1} S_{2} \dot{\theta}_{2}\right)+m_{2} g l_{2}\left(S_{3} C_{4} \dot{\theta}_{3}+C_{3} S_{4} \dot{\theta}_{4}\right) \\
&=\left(T_{\varphi}-T_{f}-D_{\varphi}\right) \dot{\varphi}+\left(F_{x}-F_{f}-D_{x}\right) \dot{x} \\
&+D_{1} \dot{\theta}_{1}+D_{2} \dot{\theta}_{2}+D_{3} \dot{\theta}_{3}+D_{4} \dot{\theta}_{4} \\
& D_{\varphi}=d_{\varphi} \dot{\varphi}, D_{x}=d_{x} \dot{x}, D_{1}=d_{1} \dot{\theta}_{1} \\
& D_{2}=d_{2} \dot{\theta}_{2}, D_{3}=d_{3} \dot{\theta}_{3}, D_{14}=d_{4} \dot{\theta}_{4} .
\end{aligned}
$$

where $D_{\ell}(\ell=\varphi, x, 1, \ldots, 4)$ is the air friction forces and $d_{\ell}$ are the damping coefficients.

Inspired by Eq. (8), combined with the control objectives of Eq. (3) and Eq. (4), construct the following positive definite functions as Lyapunov candidate functions:

$$
\begin{aligned}
V_{E} & =\frac{1}{2} \dot{q}_{e}^{T} M \dot{q}_{e}+\left(m_{1}+m_{2}\right) g l_{1}\left(1-C_{1} C_{2}\right) \\
& +m_{2} g l_{2}\left(1-C_{3} C_{4}\right)
\end{aligned}
$$

where $q_{e}=\left[\begin{array}{llllll}e_{\varphi} & e_{x} & \theta_{1} & \theta_{2} & \theta_{3} & \theta_{4}\end{array}\right]^{T}$, $\dot{q}_{e}=\left[\begin{array}{llllll}\dot{e}_{\varphi} & \dot{e}_{x} & \dot{\theta}_{1} & \dot{\theta}_{2} & \dot{\theta}_{3} & \dot{\theta}_{4}\end{array}\right]^{T}, e_{\varphi}=\varphi-\varphi_{r}, \dot{e}_{\varphi}=$ $\dot{\varphi}-\dot{\varphi}_{r}, e_{x}=x-x_{r}, \dot{e}_{x}=\dot{x}-\dot{x}_{r}$.

Then taking the time derivative of Eq. (11), we have

$$
\begin{aligned}
\dot{V}_{E} & =\dot{q}_{e}^{T} M \ddot{q}_{e}+\frac{1}{2} \dot{q}_{e}^{T} \dot{M} \dot{q}_{e}+\left(m_{1}+m_{2}\right) g l_{1}\left(S_{1} C_{2} \dot{\theta}_{1}\right. \\
& \left.+C_{1} S_{2} \dot{\theta}_{2}\right)+m_{2} g l_{2}\left(S_{3} C_{4} \dot{\theta}_{3}+C_{3} S_{4} \dot{\theta}_{4}\right) \\
& =\dot{q}_{e}^{T}\left(M \ddot{q}_{e}+C \dot{q}_{e}\right)+\left(m_{1}+m_{2}\right) g l_{1}\left(S_{1} C_{2} \dot{\theta}_{1}\right. \\
& \left.+C_{1} S_{2} \dot{\theta}_{2}\right)+m_{2} g l_{2}\left(S_{3} C_{4} \dot{\theta}_{3}+C_{3} S_{4} \dot{\theta}_{4}\right) \\
& =\dot{q}_{e}^{T}\left(U-F_{s}-D-M \ddot{q}_{\varsigma}-C \dot{q}_{\varsigma}-G\right)+\left(m_{1}\right. \\
& \left.+m_{2}\right) g l_{1}\left(S_{1} C_{2} \dot{\theta}_{1}+C_{1} S_{2} \dot{\theta}_{2}\right)+m_{2} g l_{2}\left(S_{3} C_{4} \dot{\theta}_{3}\right. \\
& \left.+C_{3} S_{4} \dot{\theta}_{4}\right) \\
& =\dot{q}_{e}^{T}\left(U-F_{s}-D-M \ddot{q}_{\varsigma}-C \dot{q}_{\varsigma}\right) \\
& =\left(T_{\varphi}-\gamma^{T}{ }_{\varphi} \varpi_{\varphi}\right) \dot{e}_{\varphi}+\left(F_{x}-\gamma^{T}{ }_{x} \varpi_{x}\right) \dot{e}_{x}+\partial_{1} \\
& +\partial_{2}+\partial_{3}+\partial_{4}
\end{aligned}
$$

where $\ddot{q}_{\varsigma}=\left[\begin{array}{llllll}\ddot{\varphi}_{r} & \ddot{x}_{r} & 0 & 0 & 0 & 0\end{array}\right]^{T}$, $\dot{q}_{\varsigma}=\left[\begin{array}{llllll}\dot{\varphi}_{r} & \dot{x}_{r} & 0 & 0 & 0 & 0\end{array}\right]^{T}, \partial_{i}(i=1, \ldots, 4)$ are included in Appendix B. 


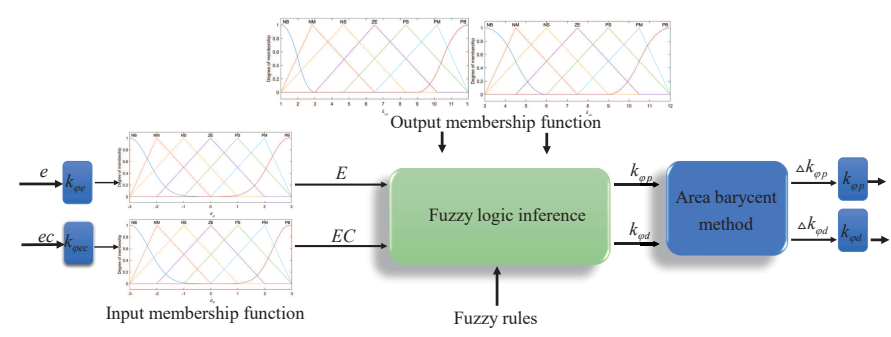

Fig. 2 Fuzzy controller structure diagram

Because the tower crane system is very complicated in the actual working environment, many parameters cannot be obtained easily, thus we estimate them in $\hat{\varpi}_{\varphi}$ and $\hat{\varpi}_{x}$, respectively, the nonlinear controller is designed as follows:

$$
\begin{aligned}
& T_{\varphi}=-k_{\varphi p} e_{\varphi}-k_{\varphi d} \dot{e}_{\varphi}+\gamma^{T}{ }_{\varphi} \hat{\varpi}_{\varphi} \\
& F_{x}=-k_{x p} e_{x}-k_{x d} \dot{e}_{x}+\gamma^{T}{ }_{x} \hat{\varpi}_{x}
\end{aligned}
$$

where in $k_{\varphi p}, k_{\varphi d}, k_{x p}, k_{x d} \in R^{+}$represent positive control gains. $\hat{\varpi}_{\varphi}$ and $\hat{\varpi}_{x}$ denote the estimations for uncertain vectors $\varpi_{\varphi}$ and $\varpi_{x}$, which are expressed as:

$$
\begin{aligned}
& \hat{\varpi}_{\varphi}=\left[\begin{array}{llllll}
\widehat{m_{1}} & \widehat{m_{2}} & \widehat{m}_{1} l_{1} & \widehat{m}_{2} l_{1} & {\widehat{m_{2} l_{2}}}^{m_{1} l_{1}^{2}}
\end{array}\right.
\end{aligned}
$$

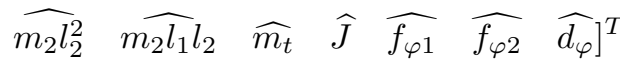

$$
\begin{aligned}
& \hat{\varpi}_{x}=\left[\begin{array}{lllllll}
\widehat{m_{1}} & \widehat{m_{2}} & \widehat{m}_{1} l_{1} & \widehat{m}_{2} l_{1} & \widehat{m}_{2} l_{2} & \widehat{m_{t}} & \widehat{f_{x 1}}
\end{array}\right. \\
& \left.\widehat{f_{x 2}} \quad \widehat{d_{x}}\right]^{T}
\end{aligned}
$$

The parameter update laws are designed as follows:

$$
\begin{aligned}
& \dot{\hat{\varpi}}_{\varphi}=-\Lambda_{\varphi}^{-1} \gamma_{\varphi}\left(\varphi-\varphi_{r}\right) \\
& \dot{\hat{\varpi}}_{x}=-\Lambda_{x}^{-1} \gamma_{x}\left(x-x_{r}\right)
\end{aligned}
$$

where $\Lambda_{\varphi}=\operatorname{diag}\left(\mu_{\varphi 1}, \mu_{\varphi 2}, \ldots, \mu_{\varphi 14}\right) \in R^{14 \times 14}, \Lambda_{x}=$ $\operatorname{diag}\left(\mu_{x 1}, \mu_{x 2}, \ldots, \mu_{x 9}\right) \in R^{9 \times 9}$ are positive definite diagonal matrices, their parameters can be designed.

\subsection{Fuzzy Controller Design}

Based on the frequency and complexity of the actual operation of the crane, in order to complete the control requirements more effectively, we added a fuzzy control method to adjust its parameters $k_{\varphi p}, k_{\varphi d}, k_{x p}$ and $k_{x d}$ online to achieve stronger real-time performance and higher control accuracy.

Two fuzzy controllers are added in the adaptive controller. Considering the similarity of the fuzzy controller in $T_{\varphi}$ and $F_{x}$, the controller with $T_{\varphi}$ in Eq. (13) is taken as an example. Taking the displacement and speed error of $\varphi$ as input, the output signals are the corrected $\Delta k_{\varphi p}$ and $\Delta k_{\varphi d}$.
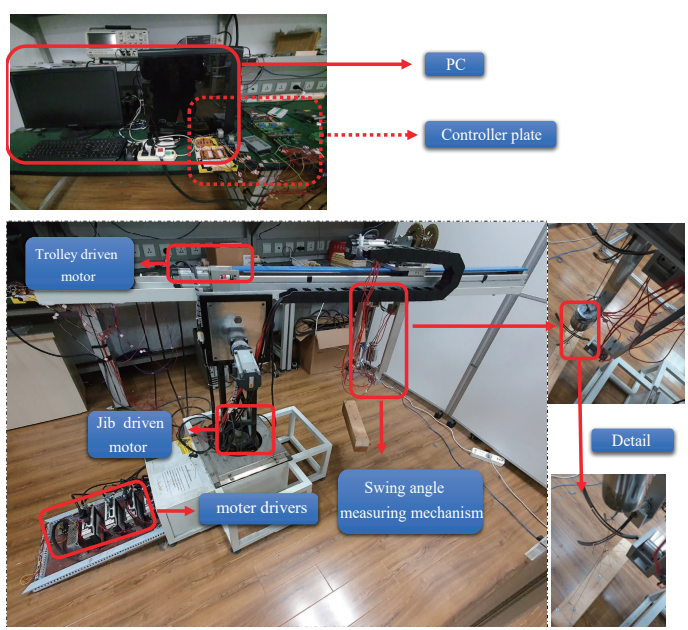

Fig. 3 Tower crane experiment platform diagram.

In Fig. 2, NB (negative big), NM (negative medium), NS (negative small), ZE (zero), PS (positive small), PM (positive medium) and PB (positive big) are chosen for $e_{\varphi}, \dot{e}_{\varphi}, \Delta k_{\varphi p}$ and $\Delta k_{\varphi d}$. Among them, The basic domain of the jib displacement and velocity errors are all $\varphi \in[-3,3]$. The basic domains of revision are $\Delta k_{\varphi p} \in[1,12]$ and $\Delta k_{\varphi d} \in[3,12]$. Their quantization factors are $0.25,20,10$ and 10 respectively and use trimf and gaussmf type functions as membership functions. According to the rules in Fig. 3, the adaptive adjustment of $k_{\varphi p}$ and $k_{\varphi d}$ parameters are:

$k_{\varphi p}=k_{\varphi p}^{0}+\Delta k_{\varphi p}$

$k_{\varphi d}=k_{\varphi d}^{0}+\Delta k_{\varphi d}$

where $k_{\varphi p}^{0}$ and $k_{\varphi d}^{0}$ are the initial values of the controller parameter, and $\Delta k_{\varphi p}, \Delta k_{\varphi d}$ are the two modified values.

The fuzzy rules for tuning the control gains are expressed generally as:

If $E$ is $M_{i 1}$ and $E C$ is $M_{j 1}$ then $k_{\varphi p}$ is $Q_{i 1}$ and $k_{\varphi d}$ is $Q_{j} 1$ )

where $M_{i 1}, M_{j 1}, Q_{i 1}$ and $Q_{j 1}$ represent different membership curves. In addition, set two inputs and seven fuzzy sets, the total rule is forty nine, as given in Fig. 3 .

\subsection{Stability Analysis}

Theorem 1: Under the controller and update laws Eqs. (13)-(18), the trolley and jib track the reference trajectories and the swing of the hook and DMP will be 
suppressed, that is:

$$
\begin{aligned}
& {\left[\begin{array}{ll}
e_{\varphi} & e_{x}
\end{array}\right] \in e_{\chi}{ }^{*}, \quad\left[\begin{array}{llll}
\theta_{1} & \theta_{2} & \theta_{3} & \theta_{4}
\end{array}\right] \in \theta_{\iota}{ }^{*}} \\
& \lim _{x \rightarrow \infty}\left[\begin{array}{ll}
e_{\chi}{ }^{*} & \theta_{\iota}{ }^{*}
\end{array}\right]^{T}=\left[\begin{array}{llllll}
0 & 0 & 0 & 0 & 0 & 0
\end{array}\right]^{T} \\
& \lim _{x \rightarrow \infty}\left[\begin{array}{cc}
\dot{e}_{\chi}^{*} & \dot{\theta}_{\iota}^{*}
\end{array}\right]^{T}=\left[\begin{array}{llllll}
0 & 0 & 0 & 0 & 0 & 0
\end{array}\right]^{T} \\
& \lim _{x \rightarrow \infty}\left[\begin{array}{ll}
\ddot{e}_{\chi}^{*} & \ddot{\theta}_{\iota}^{*}
\end{array}\right]^{T}=\left[\begin{array}{llllll}
0 & 0 & 0 & 0 & 0 & 0
\end{array}\right]^{T}
\end{aligned}
$$

Proof: See the Appendix C.

\section{Experimental Results And Discussions}

This section will be divided into three parts. The first part is the introduction of the self-built tower crane experiment platform, the second part presents experimental conditions, and the third part is the comparative experiment and analysis with the existing controllers. The comparative controllers are chosen linearquadratic-regulator (LQR) [25] and partial enhancedcoupling (PEC) [26].

\subsection{Experimental Equipment}

The tower crane platform is shown in Fig. 4. The platform consists of PC, control plate, trolley, jib, motor driver and swing angle measuring mechanisms. In terms of specific operation, use Matlab/Simulink to build and generate code on the PC, and send it to the Digital Signal Processing (DSP) with a control cycle of $5[\mathrm{~ms}]$, and then monitor and record feedback through the serial port data. It is worth mentioned that the four swing angle measuring mechanisms are used to monitor the swing of the hook and DMP (the details are shown in Fig. 4).

\subsection{Experimental Conditions}

In order to avoid the adverse effects of too fast or too slow positioning of the control process, and to meet the preset control target, the following reference trajectories are adopted:

$q(\chi)_{r}=\left\{\begin{array}{rr}\left(\frac{t}{t_{q(\chi)_{d}}}-\frac{\sin \left(\frac{2 \pi t}{t_{q(\chi)_{d}}}\right)}{2 \pi}\right)\left(q(\chi)_{d}-q(\chi)_{0}\right. \\ +q(\chi)_{0}, t \in\left[0, t_{q(\chi)_{d}}\right) \\ q(\chi)_{d} . & t \in\left[t_{q(\chi)_{d}},+\infty\right)\end{array}\right.$

where $q(\chi)_{0}, q(\chi)_{d}$ and $t_{q(\chi) d}$ respectively represent the initial target position/angle, the final target position/angle, and the time required to reach. In addition, $q(\chi)_{0}=$ $0[\mathrm{~m}], t_{q(\chi) d}=3[\mathrm{~s}], q(\varphi)_{r}=\varphi_{r}=-30[\mathrm{deg}], q(x)_{r}=$ $x_{r}=0.3[\mathrm{~m}]$. Model parameters are shown in TABLE I.

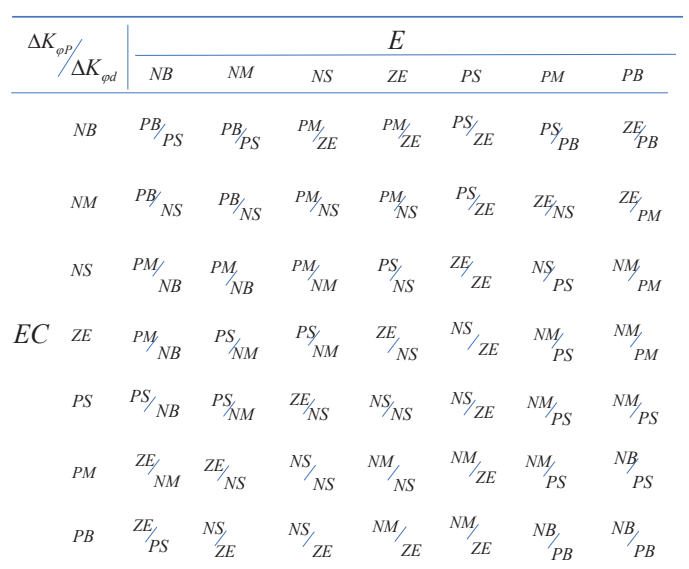

Fig. 4 Fuzzy control rules.

Table 1 Tower crane model

\begin{tabular}{|c|c|c|c|c|c|}
\hline$m_{t}[\mathrm{Kg}]$ & 3.0 & $m_{1}[\mathrm{Kg}]$ & 0.5 & $m_{2}[\mathrm{Kg}]$ & 0.5 \\
\hline$l_{1}[\mathrm{~m}]$ & 0.4 & $J_{0}[\mathrm{Nm}]$ & 6.8 & $g\left[\mathrm{~m} / s^{2}\right]$ & 9.80 \\
\hline
\end{tabular}

According to the time response of the crane system, we choose the adaptive laws $\Lambda_{\varphi}$ and $\Lambda_{x}$ to be as 0.02 and 10, respectively. The initial fuzzy controller gains of $T_{\varphi}$ and $F_{x}$ are:

$k_{\varphi p}^{0}=20, k_{\varphi d}^{0}=5, k_{x p}^{0}=10, k_{x d}^{0}=5$

\subsection{Existing Controller}

In this section, the proposed controller is compared with the existing controllers (LQR and PEC) for experimental comparison and analysis. First, linearize the model for the following controller design.

$M^{*} \ddot{q}+G^{*}(q)=U$

where

$M^{*}=\left[m^{\prime}{ }_{i j}\right] \in R^{6 \times 6}$.

$i=1, \ldots, 6, j=1, \ldots, 6$.

and $m_{11}^{*}=J+\left(m_{1}+m_{2}+m_{t}\right) x_{d}^{2}, m_{22}^{*}=m_{1}+m_{2}+$ $m_{t}, m_{33}^{*}=\left(m_{1}+m_{2}\right) l_{1}^{2}, m_{44}^{*}=\left(m_{1}+m_{2}\right) l_{1}^{2}, m_{55}^{*}=$ $m_{2} l_{p} / 12+m_{2} l_{2}^{2}, m_{66}^{*}=m_{2} l_{2}^{2}, m_{16}^{*}=m_{61}^{*}=-m_{2} l_{2} x_{d}$, $m_{14}^{*}=m_{41}^{*}=-\left(m_{1}+m_{2}\right) l_{1} x_{d}, m_{23}^{*}=m_{32}^{*}=\left(m_{1}+\right.$ $\left.m_{2}\right) l_{1}, m_{25}^{*}=m_{52}^{*}=m_{2} l_{2}, m_{35}^{*}=m_{53}^{*}=m_{2} l_{1} l_{2}, m_{46}^{*}=$ $m_{64}^{*}=m_{2} l_{1} l_{2}$. The rest of the elements are all zero.

$G^{*}(q)=\left[\begin{array}{llllll}g_{1}^{*} & g_{2}^{*} & g_{3}^{*} & g_{4}^{*} & g_{5}^{*} & g_{6}^{*}\end{array}\right]^{T}$

where $g_{1}^{*}=0, g_{2}^{*}=0, g_{3}^{*}=\left(m_{1}+m_{2}\right) l_{1} g \theta_{1}, g_{4}^{*}=$ $\left(m_{1}+m_{2}\right) l_{1} g \theta_{2}, g_{5}^{*}=l_{2} m_{2} g \theta_{3}, g_{6}^{*}=l_{2} m_{2} g \theta_{4}$.

$U=\left[\begin{array}{llllll}T_{\varphi} & F_{x} & 0 & 0 & 0 & 0\end{array}\right]^{T}$. 
Then, the first controller LQR based on Eq. (25) is designed as follows:

$$
\begin{aligned}
T_{\varphi}^{l q r} & =-k_{\varphi 1} e_{\varphi}-k_{\varphi 2} \dot{e}_{\varphi}-k_{\varphi 3} \theta_{2} \\
& -k_{\varphi 4} \dot{\theta}_{2}-k_{\varphi 5} \theta_{4}-k_{\varphi 6} \dot{\theta}_{4}+T_{f} \\
F_{x}^{l q r} & =-k_{x 1} e_{x}-k_{x 2} \dot{e}_{x}-k_{x 3} \theta_{1} \\
& -k_{x 4} \dot{\theta}_{1}-k_{x 5} \theta_{3}-k_{x 6} \dot{\theta}_{3}+F_{f}
\end{aligned}
$$

where $T_{f}$ and $F_{f}$ are friction forces in Appendix B. Through multiple experiments and analysis, the friction parameters are selected as $f_{\varphi 1}=2.2, f_{\varphi 2}=0.8, f_{x 1}=$ $0.5, f_{x 2}=0.5, \varepsilon_{1}=0.01, \varepsilon_{2}=0.01$. Then, the gain parameters of the controller as follows:

$$
\begin{aligned}
& k_{\varphi 1}=56.6, k_{\varphi 2}=14.5, k_{\varphi 3}=8.9, \\
& k_{\varphi 4}=3.4, k_{\varphi 5}=-2.5, k_{\varphi 6}=-1.1, \\
& k_{x 1}=40.0, k_{x 2}=9.2, k_{x 3}=-19.1, \\
& k_{x 4}=-0.9, k_{x 5}=13.3, k_{x 6}=0.8 .
\end{aligned}
$$

The second controller PEC is designed as follows:

$$
\begin{aligned}
T_{\varphi}^{p e c} & =-k_{11} e_{\vartheta_{\varphi}}-k_{12} \dot{e}_{\vartheta_{\varphi}}-k_{1}\left(\dot{\theta}_{2}^{2}+\dot{\theta}_{4}^{2}\right) \dot{e}_{\vartheta_{\varphi}} \\
& +m_{11}^{*}\left(\eta_{\varphi}\left(m_{41}^{*} \dot{\theta}_{2}+m_{61}^{*} \dot{\theta}_{4}\right)+\ddot{\varphi}_{r}\right)+T_{f} \\
F_{x}^{\text {pec }} & =-k_{21} e_{\vartheta_{x}}-k_{22} \dot{e}_{\vartheta_{x}}-k_{2}\left(\dot{\theta}_{1}^{2}+\dot{\theta}_{3}^{2}\right) \dot{e}_{\vartheta_{x}} \\
& +m_{22}^{*}\left(\eta_{x}\left(m_{32}^{*} \dot{\theta}_{1}+m_{52}^{*} \dot{\theta}_{3}\right)+\ddot{x}_{r}\right)+F_{f}
\end{aligned}
$$

where

$e_{\vartheta_{\varphi}}=\varphi(t)-\int_{0}^{t} \eta_{\varphi} m^{*}{ }_{41} \theta_{2}+\eta_{\varphi} m^{*}{ }_{61} \theta_{4} d \tau-\varphi_{r}$

$e_{\vartheta_{x}}=x(t)-\int_{0}^{t} \eta_{x} m^{*}{ }_{32} \theta_{1}+\eta_{x} m^{*}{ }_{52} \theta_{3} d \tau-x_{r}$

and $m_{11}^{*}, m_{41}^{*}, m_{61}^{*}, m_{22}^{*}, m_{32}^{*}$ and $m_{52}^{*}$ are the linearization variables of the system. Besides, the parameters of the controller are selected as follows [26]:

$k_{11}=80, k_{12}=15, k_{1}=10, k_{21}=150$,

$k_{22}=10, k_{2}=10, \eta_{\varphi}=0.8, \eta_{x}=0.51$.

\subsection{Peformance Comparison}

We let the three controllers follow the target trajectories in Eq. (23). The experimental comparison results are shown in Fig. 5, which are analyzed and compared from three aspects. The first one is for positioning accuracy. Compared with the proposed control, within $0[\mathrm{~s}]-$ $2[\mathrm{~s}], \mathrm{PEC}$ and LQR controllers show hysteresis effects, and within $3[\mathrm{~s}]-4[\mathrm{~s}]$, PEC and LQR show leading effect$\mathrm{s}$, which is very easy to produce overshoot. Beside, the contrast controllers did not reach the target position in the rotation angle (see the enlarged image for details). The second one is for the swing suppression time. The proposed controller completes sway elimination in $6[\mathrm{~s}]$.

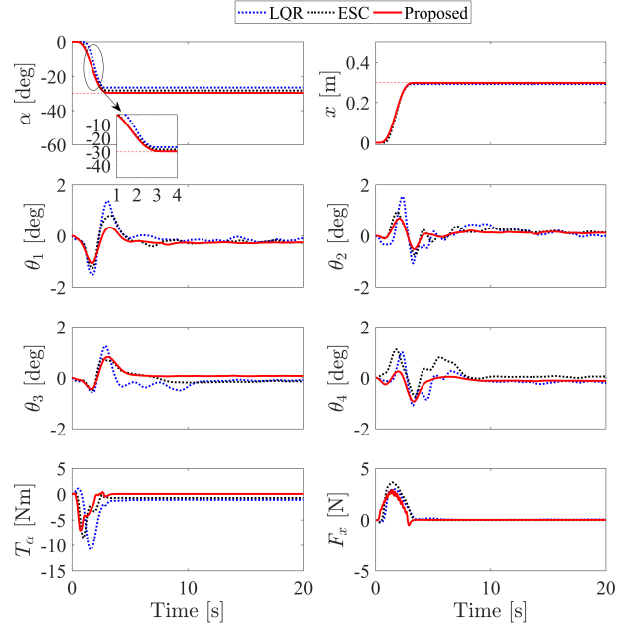

Fig. 5 Results of experiment A: $m_{2}=0.5 \mathrm{~kg}, l_{2}=0.15 \mathrm{~m}$.

Table 2 Experimental results with $m_{2}=0.5[\mathrm{~kg}], l_{h}=0.15$ [m]

\begin{tabular}{|c|l|l|l|}
\hline Control method & $t_{\alpha r}[\mathrm{~s}]$ & $t_{x r}[\mathrm{~s}]$ & $\theta_{1 \max }[\mathrm{deg}]$ \\
\hline Proposed controller & 3.04 & 3.00 & 1.01 \\
\hline PEC & 2.97 & 3.03 & 1.26 \\
\hline LQR & 2.82 & 3.00 & 1.37 \\
\hline & $\theta_{2 \max }[\mathrm{deg}]$ & $\theta_{3 \max }[\mathrm{deg}]$ & $\theta_{4 \max }[\mathrm{deg}]$ \\
\hline Proposed controller & 0.67 & 0.81 & 0.92 \\
\hline PEC & 0.93 & 0.72 & 1.10 \\
\hline LQR & 1.07 & 1.25 & 1.03 \\
\hline
\end{tabular}

Nevertheless, PEC and LQR complete the swing elimination at $10[\mathrm{~s}]$. The third one is for the maximum swing angles. The overall swing of the proposed controller is smaller than that of the comparison controllers. The specific content is shown in TABLE II.

\subsection{Robustness Verification}

This section will verify the robustness of the proposed controller from two parts.

In the first part, by changing the mass/rope length of the DMP, the mass $m_{2}$ and the rope length $l_{2}$ are respectively $0.5[\mathrm{~kg}]-1.5[\mathrm{~kg}]$ and $0.15[\mathrm{~m}]-0.25[\mathrm{~m}]$. In Fig. 6 and Fig. 7, we analyze from two directions. In the aspect of anti-swing, the proposed controller can complete the anti-swing within $6.5[\mathrm{~s}]$. However, the comparison controllers PEC and LQR generally extend $4[\mathrm{~s}]-6[\mathrm{~s}]$ for the sway time, and the sway elimination is completed in about $14[\mathrm{~s}]-16[\mathrm{~s}]$. Moreover, the maximum swing angles obtained by the proposed controller is still smaller 


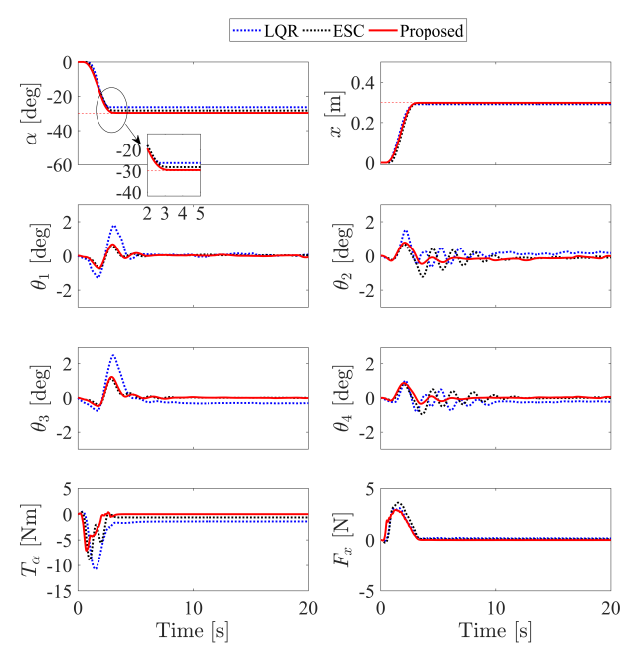

Fig. 6 Results of experiment B: $m_{2}=1 \mathrm{~kg}, l_{2}=0.2 \mathrm{~m}$.

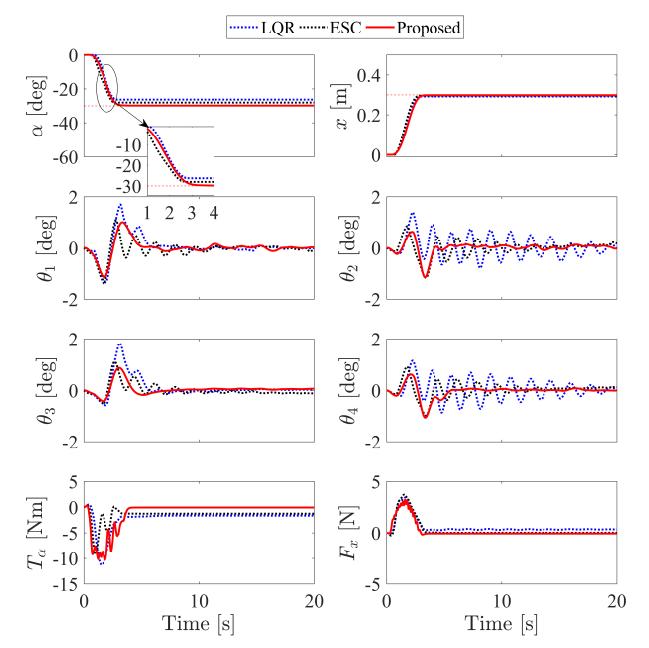

Fig. 7 Results of experiment C: $m_{2}=1.5 \mathrm{~kg}, l_{2}=0.25 \mathrm{~m}$.

than that obtained by the comparison controllers. It can be concluded that under different parameter changes, the proposed controller still has good performance. In contrast, PEC and LQR performed poorly. The main reason is that the contrast controllers require a higher model accuracy. Therefore, this makes performance of the contrast controllers change drastically.

In the second part, considering the payload swing caused by loading goods, a non-zero initial swing angle experiment was further carried out. Specifically, the initial angles of $\theta_{i}(i=1 \cdots 4)$ under the proposed controller were set as $-0.5[\mathrm{deg}],-0.4[\mathrm{deg}], 0.2[\mathrm{deg}]$ and $-0.1[\mathrm{deg}]$, and the corresponding values of PEC and LQR were set as $-0.1[\mathrm{deg}],-0.5[\mathrm{deg}],-0.2[\mathrm{deg}],-0.3[\mathrm{deg}$, $-0.1[\mathrm{deg}],-0.8[\mathrm{deg}],-0.1[\mathrm{deg}]$ and $-0.4[\mathrm{deg}]$, respectively. The results are shown in Fig. 8. Due to the different initial angles, we only study the problem of posi-

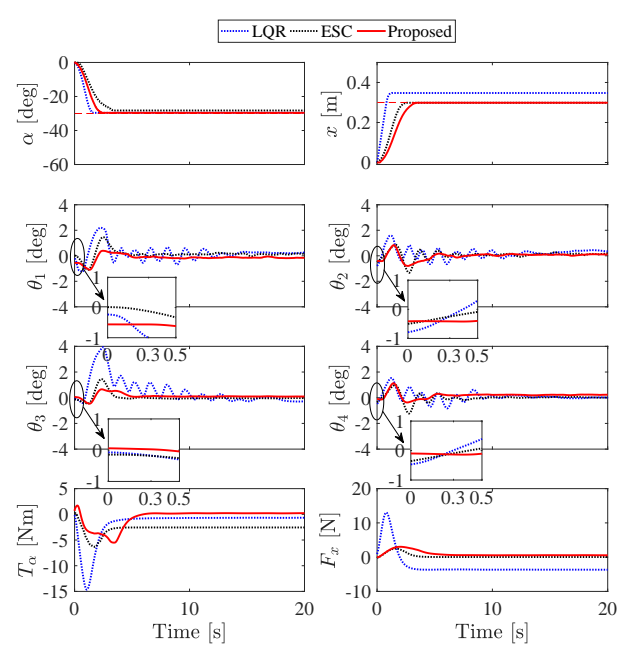

Fig. 8 Non-zero initial swing angle experiment D: $m_{2}=$ $1.0 \mathrm{~kg}, l_{2}=0.2 \mathrm{~m}$.

tioning and eliminating swing. In terms of positioning accuracy, the fuzzy controller online adjustment avoids the failure of the LQR positioning. At the same time, it also avoids the large swing angles caused by the PEC lagging in the $\varphi$ direction and the leading in the $x$ direction. In terms of swing suppression, the LQR controller has the worst performance ( the payload swing angles are suppressed in 16[s].), followed by PEC ( the antiswing effect of $\theta_{1}$ and $\theta_{3}$ are better, while the effect of $\theta_{2}$ and $\theta_{4}$ are poor ). In contrast, it is not difficult to find that the proposed controller still has a greater advantage.

\section{Conclusion}

In this paper, a fuzzy adaptive controller was designed for the controlling goal of DTCDMP. The proposed controller consists of two parts: the adaptive controller and the fuzzy controller. This paper first given the expression of system energy. According to the expression of system energy, a scalar function was proposed, and an adaptive controller was designed based on this. Then, considering the real-time control of actual operation, we added fuzzy control to further improve its positioning accuracy and anti-sway function. Finally, a large num, ber of comparative experiments shown that the proposed controller still has excellent performance regardless of the uncertain model parameters, non-zero initial angles and external disturbances. 


\section{Acknowledgment}

This work is supported in part by the National Natural Science Foundation of China under Grant 61703202.

\section{Conflict of Interests}

The authors declare that there is no conflict of interests regarding the publication of this article.

\section{Data Availability Statements}

The datasets generated and analyzed during the current study are available from the corresponding author on reasonable request.

\section{References}

1. Fu Y, Sun N, Yang T, Qiu Z, Fang Y. Adaptive coupling anti-swing tracking control of underactuated dual boom crane systems. IEEE Transactions on Systems Man and Cybernetics Systems in press 2021.

2. Liu Y, Mei Y, Cai H, He C, Liu T, Hu G. Asymmetric input-output constraint control of a flexible variablelength rotary crane arm. IEEE Transactions on Cybernetics in press 2021.

3. Mohammad MJ, Mohamed Z, Sudin, Buyamin S, Jaafar HI, Ahmad SM. An improved input shaping design for an efficient sway control of a nonlinear 3D overhead crane with friction. Mechanical Systems and Signal Processing 2017; 92: 364-378.

4. Potter J, Singhose W. Design and human-in-the-loop testing of reduced-modification input shapers. IEEE Transactions on Control Systems Technology 2016; 24(4): 1513-1520.

5. Xie X, Huang J, Liang Z. Vibration reduction for flexible systems by command smoothing. Mechanical Systems and Signal Processing 2013; 39(1-2): 461-470.

6. Li X, Geng Z. A novel trajectory planning-based adaptive control method for 3-D overhead cranes. International Journal of Systems Science 2018; 9(16): 3332-3345.

7. Zhang X, Fang Y, Sun N. Minimum-time trajectory planning for underactuated overhead crane systems with state and control constraints. IEEE Transactions on Industrial Electronics 2014; 61(12): 6915-6925.

8. Le TA, Lee SG, Moon SC. Partial feedback linearization and sliding mode techniques for $2 \mathrm{D}$ crane control. Transactions of the Institute of Measurement and Control 2014; 36 (1): 78-87.

9. Sun N, Fang Y, New energy analytical results for the regulation of underactuated overhead cranes: an end-effector motion-based approach. IEEE Transactions on Industrial Electronics 2012; 59(12): 4723-4734.

10. $\mathrm{He} \mathrm{W}, \mathrm{Ge} \mathrm{S}$. Cooperative control of a nonuniform gantry crane with constrained tension. Automatica 2016; 66: 146-154.

11. Sun N, Yang T, Chen H, Fang Y, Qian Y. Adaptive antiswing and positioning control for 4-DOF rotary cranes subject to uncertain/unknown parameters with hardware experiments. IEEE Transactions on Systems Man and Cybernetics Systems 2017; 49(7): 1309-1321.
12. Sun N, Fang Y, Chen H. A new antiswing control method for underactuated cranes with unmodeled uncertainties: theoretical design and hardware experiments. IEEE Transactions on Industrial Electronics 2015; 62(1): 453-465.

13. Tuan L, Cuong H, Trieu P, Nho L, Thuan V, Anh L. Adaptive neural network sliding mode control of shipboard container cranes considering actuator backlash. Mechanical Systems and Signal Processing 2018; 112: 233-250.

14. Park M, Chwa D, Eom M. Adaptive sliding-mode antisway control of uncertain overhead cranes with highspeed hoisting motion. IEEE Transactions on Fuzzy Systems 2014; 22(5): 1262-1271.

15. Lee L, Huang P, Shih Y, Chiang T, Chang C. Parallel neural network combined with sliding mode control in overhead crane control system. Journal of Vibration and Control 2012; 20(5): 749-760.

16. Qian Y, Hu D, Chen Y, Fang Y, Hu Y. Adaptive neural network-based tracking control of underactuated offshore ship-to-ship crane systems subject to unknown wave motions disturbances. IEEE Transactions on Systems Man and Cybernetics Systems in press 2021.

17. Yang T, Sun N, Chen H, Fang Y. Neural network-based adaptive antiswing control of an underactuated shipmounted crane with roll motions and input dead zones. IEEE Transactions on Neural Networks and Learning Systems 2020; 31(3): 901-914

18. Jaafar H, Mohamed Z, Shamsudin M, Mohd S, Liyana R, Abdullahi A. Model reference command shaping for vibration control of multimode flexible systems with application to a double-pendulum overhead crane. Mechanical Systems and Signal Processing 2019; 115: 677-695.

19. Lu B, Fang Y, Sun N. Enhanced-coupling adaptive control for double-pendulum overhead cranes with payload hoisting and lowering. Automatica 2019; 101: 241-251.

20. Ouyang H, Tian Z, Yu L, Zhang G. Load swing rejection for double-pendulum tower cranes using energy-shapingbased control with actuator output limitation. ISA Transactions 2020; 101: 246-255.

21. Giacomelli M, Padula F, Simoni L, Visioli A. Simplified input-output inversion control of a double-pendulum overhead crane for residual oscillations reduction. Mechatronics 2018, 56: 37-47.

22. Sun N, Wu Y, Chen H, Fang Y. An energy-optimal solution for transportation control of cranes with doublependulum dynamics: design and experiments.Mechanical Systems and Signal Processing 2018; 102: 87-101.

23. Chen H, Fang Y, Sun N. A swing constrained time-optimal trajectory planning strategy for doublependulum crane systems. Nonlinear Dynamics 2017; 89: $1513-1524$

24. Wu Q, Wang X, Hua L, Xia M. Improved time optimal anti-swing control system based on low-pass filter for double-pendulum crane system with distributed mass beam. Mechanical Systems and Signal Processing 2020; 151: 107-444.

25. Scampicchio A, Aravkin A, Pillonetto G. Stable and robust LQR design via scenario approach. Automatica 2021; 129: 109-571.

26. Ouyang H, Tian Z, Yu L, Zhang G. Partial enhancedcoupling control approach for trajectory tracking and swing rejection in tower cranes with double-pendulum effect. Mechanical Systems and Signal Processing 2021; 156: 107-613. 